\title{
SOLUTIONS OF SEVERAL PROBLEMS IN THE THEORY OF COMPACT POSITIVE OPERATORS
}

\author{
Y. A. ABRAMOVICH AND A. W. WICKSTEAD
}

(Communicated by Palle E. T. Jorgensen)

\begin{abstract}
We construct a compactly dominated compact operator $S$ on a Dedekind complete Banach lattice whose modulus $|S|$ is not compact. We also construct a compactly dominated compact operator without modulus.
\end{abstract}

\section{INTRODUCTION}

In his work $[\mathrm{K} 1,2]$ Krengel showed that a compact operator $S$ on a Banach lattice $E$ may fail to have a modulus, and also that the modulus (if it exists) may fail to be compact. In our work [AW] we strengthened Krengel's example by showing that even an extra assumption that $S$ is regular (i.e., $S \leq U$ for some positive operator $U$ ) does not imply the existence of the modulus. Our investigation was motivated (at least partly) by a common belief that there should exist some nice relationships between compactness and order properties of operators on Banach lattices. In particular, in our quest for such properties we posed the following questions:

(Q1) Let $S: E \rightarrow E$ be a compact operator on a Banach lattice and let $S$ be dominated by a compact positive operator $U$, i.e., $|S x| \leq U x$ for each $x \geq 0$. Does this imply that $S$ has a modulus?

(Q2) If (under the above conditions) the modulus $|S|$ does exist, must it be compact?

The same questions were repeated in the problem section in [HL, Problem 3]. The purpose of this article is to answer (in the negative) these questions.

It is worth pointing out that problem $(\mathrm{Q} 1)$ is nontrivial only for non-Dedekind complete Banach lattices (since otherwise $|S|$ exists by the Riesz-Kantorovich theorem), while problem (Q2) is nontrivial for any Banach lattice. It has long been known that if $E$ and $E^{\prime}$ have order continuous norms, then the DoddsFremlin theorem [DF] shows that the compactly dominated regular operators are all compact and form a lattice ideal in the Dedekind complete vector lattice of all regular operators on $E$, so the answer to $\left(\mathrm{Q}^{2}\right)$ is certainly positive in this

Received by the editors February 28, 1994.

1991 Mathematics Subject Classification. Primary 47B60, 47B06; Secondary 46B42, 46A50.

Key words and phrases. Banach lattice, compact operator, modulus.

This work was made possible by a NATO Collaborative Research Grant CRG-890909 which allowed Prof. Wickstead to visit IUPUI during the summer of 1993. 
case. In [W1] the second author has characterized pairs of Banach lattices $E, F$ such that compactly dominated operators from $E$ into $F$ are compact. Obviously, the answer to $(\mathrm{Q} 2)$ is still positive in such cases. In our counterexample to (Q2) $E=F$ will be Dedekind complete, so by this example the question (Q2) is closed completely. Moreover, this example also allows us to solve the following well-known and rather old question posed by C. D. Aliprantis and O. Burkinshaw in 1982 at a Riesz Spaces and Operator Theory meeting at Oberwolfach (see also [HL, Problem 6]).

(Q3) Let $K_{1}, K_{2}$ be two positive compact operators on a Dedekind complete Banach lattice $E$. Must their supremum $K_{1} \vee K_{2}$ also be compact?

In Corollary 2 this question will be answered in the negative. For terminology and notation not explained below we refer to $[\mathrm{AB}]$.

\section{Counterexamples to (Q2) AND (Q3)}

The central idea of Krengel's (and, essentially, of all subsequent) examples (see for instance $\left[\mathrm{AB}\right.$, p. 270]) is that on $2^{n}$-dimensional Euclidean space $l_{2}^{2^{n}}$ there is an operator $S_{n}$ such that $\left\|S_{n}\right\|=2^{n / 2}$ and $\left\|\left|S_{n}\right|\right\|=2^{n}$. Moreover (and this will be used repeatedly by us) if we use the Rademacher functions to define the matrix of the operator $S_{n}$, then all the entries of the matrix of the operator $\left|S_{n}\right|$ are equal to 1 . Therefore, $\left|S_{n}\right|$ has rank one and for each $\mathbf{x}=\left(x_{1}, \ldots, x_{2^{n}}\right) \in l_{2}^{2^{n}}$ we have $\left|S_{n}\right| \mathbf{x}=\left(\sum_{k=1}^{2^{n}} x_{k}\right) \mathbf{1}_{n}$, where

$$
\mathbf{1}_{n}=\overbrace{(1,1, \ldots, 1)}^{2^{n} \text { times }} \text {. }
$$

It will be convenient to introduce the following operator $J_{n}$ embedding $l_{2}^{2^{n}}$ into $L_{2}[0,1]$ :

$$
J_{n}: \mathbf{x}=\left(x_{1}, \ldots, x_{2^{n}}\right) \mapsto \sum_{k=1}^{2^{n}} x_{k x_{1 \frac{k-1}{2^{n}}, \frac{k}{2^{n}}}} .
$$

It is obvious that $J_{n}$ is a lattice isomorphism (i.e., $\left|J_{n} \mathbf{x}\right|=J_{n}|\mathbf{x}|$ ) and

$$
\left\|J_{n} \mathbf{x}\right\|_{L_{2}}=\left(\sum_{k} 2^{-n} x_{k}^{2}\right)^{1 / 2}=2^{-n / 2}\left(\sum_{k} x_{k}^{2}\right)^{1 / 2}=2^{-n / 2}\|\mathbf{x}\|_{l_{2}^{n}} .
$$

Thus, $\left\|J_{n} \circ S_{n}\right\|=1$ and $\left\|\left|J_{n} \circ S_{n}\right|\right\|=\left\|J_{n} \circ\left|S_{n}\right|\right\|=2^{n / 2}$.

Since $J_{n} \mathbf{1}_{\mathbf{n}}=\chi_{[0,1]}$, it follows that for each element $\mathbf{x}=\left(x_{1}, \ldots, x_{2^{n}}\right) \in l_{2}^{2^{n}}$ we have $J_{n} \circ\left|S_{n}\right| \mathbf{x}=\left(\sum_{k=1}^{2^{n}} x_{k}\right) \chi_{[0,1]}$.

We denote by $l_{1}\left(l_{2}^{n}\right)$ the $l_{1}$-direct sum of spaces $l_{2}^{2^{n}}$, i.e., $\overline{\mathbf{x}}=\left(\mathbf{x}_{1}, \mathbf{x}_{2}, \ldots\right) \epsilon$ $l_{1}\left(l_{2}^{2^{n}}\right)$ whenever $\mathbf{x}_{\mathbf{n}} \in l_{2}^{2^{n}} \quad(n \in \mathbb{N})$ and

$$
\|\overline{\mathbf{x}}\|=\sum_{n}\left\|\mathbf{x}_{\mathbf{n}}\right\|_{l_{2}^{n}}<\infty .
$$

We will be using the notation we have just introduced throughout this paper.

Definition. An operator $T: E \rightarrow F$ between Banach lattices is said to be compactly dominated if there exists a positive compact operator $U: E \rightarrow F$ satisfying $|T x| \leq U x$ for each $0 \leq x \in E$. This relationship between $T$ and $U$ can be expressed in terms of the operator order as $-U \leq T \leq U$ or $\pm T \leq U$. 
Theorem 1. (i) There exists a compactly dominated compact operator $S: l_{1}\left(l_{2}^{2^{n}}\right)$ $\rightarrow L_{2}[0,1]$, such that $|S|$ is not compact.

(ii) There exists a Dedekind complete Banach lattice $E$ and compactly dominated compact operator $S: E \rightarrow E$ such that $|S|$ is not compact.

Proof. (i) Since the order intervals in $L_{2}[0,1]$ are not norm compact, there exists a sequence $\left\{g_{n}\right\} \in L_{2}[0,1]$ satisfying $0 \leq g_{n} \leq \chi_{[0,1]}$ and with no norm-convergent subsequence. For $\overline{\mathbf{x}}=\left(\mathbf{x}_{1}, \mathbf{x}_{2}, \ldots\right) \in l_{1}\left(l_{2}^{2^{n}}\right)$ let

$$
S \overline{\mathbf{x}}=\sum_{n} 2^{-n / 2} g_{n} J_{n} S_{n} \mathbf{x}_{\mathbf{n}}
$$

As $\left\|2^{-n / 2} g_{n} J_{n} S_{n}\right\|_{L_{2}} \leq 2^{-n / 2}\left\|J_{n} \circ S_{n}\right\|=2^{-n / 2}$, the series $\sum_{n} 2^{-n / 2} g_{n} J_{n} S_{n}$, defining the operator $S$, converges absolutely, and hence $S$ is a compact operator from $l_{1}\left(l_{2}^{2^{n}}\right)$ to $L_{2}[0,1]$.

Let also

$$
U(\overline{\mathbf{x}})=\sum_{n} 2^{-n / 2} J_{n}\left|S_{n}\right| \mathbf{x}_{\mathbf{n}}
$$

The operator $U$ is well defined as

$$
\sum_{n=1}^{\infty}\left\|2^{-n / 2} J_{n} \circ\left|S_{n}\right|\left(\mathbf{x}_{\mathbf{n}}\right)\right\| \leq \sum_{n=1}^{\infty} 2^{-n / 2}\left\|J_{n} \circ\left|S_{n}\right|\right\| \cdot\left\|\mathbf{x}_{\mathbf{n}}\right\|_{l_{2}^{2^{n}}}=\sum_{n=1}^{\infty}\left\|\mathbf{x}_{\mathbf{n}}\right\|_{l_{2}^{n}}=\|\overline{\mathbf{x}}\| .
$$

The properties of $\left|S_{n}\right|$ imply that $U$ has rank 1 , and consequently $U$ is compact. A direct verification shows that $\pm S \leq U$, that is, $S$ is compactly dominated.

Since $L_{2}[0,1]$ is Dedekind complete, the modulus $|S|$ does exist. Restricting $|S|$ to $l_{2}^{2^{n}}$, which is an order ideal in $l_{1}\left(l_{2}^{n}\right)$, we obviously have

$$
|S|_{\mid l_{2}^{n}}=\left|2^{-n / 2} g_{n} \cdot J_{n} \circ S_{n}\right|=2^{-n / 2} g_{n}\left|J_{n} \circ S_{n}\right|=2^{-n / 2} g_{n} \cdot J_{n} \circ\left|S_{n}\right| \text {, }
$$

so

$$
|S|(\overline{\mathbf{x}})=\sum_{n} 2^{-n / 2} g_{n} \cdot\left(J_{n} \circ\left|S_{n}\right|\right) \mathbf{x}_{\mathbf{n}}
$$

Let $\mathbf{x}_{\mathbf{n}}=\overbrace{\left(2^{-n / 2}, 2^{-n / 2}, \ldots, 2^{-n / 2}\right)}^{2^{n} \text { times }} \in l_{2}^{2^{n}}$ and take $\overline{\mathbf{x}}=\left(0, \ldots, 0, \mathbf{x}_{\mathbf{n}}, 0, \ldots\right) \in$ $l_{1}\left(l_{2}^{2^{n}}\right)$.

Clearly, $\|\overline{\mathbf{x}}\|=1$ and $\left|S_{n}\right| \mathbf{x}_{\mathbf{n}}=2^{n / 2} \mathbf{1}_{\mathbf{n}}$, implying that $|S|(\overline{\mathbf{x}})=g_{n}$. Thus, the image under $|S|$ of the unit ball in $l_{1}\left(l_{2}^{2^{n}}\right)$ contains every function $g_{n}$. Since the sequence $\left\{g_{n}\right\}$ has no norm convergent subsequence, we see that $|S|$ is not compact, and we are done.

(ii) Having $S$ as above, it is a trivial matter to produce the desired endomorphism. Let $E:=l_{1}\left(l_{2}^{2^{n}}\right) \oplus L_{2}[0,1]$ and define $S_{1}: E \rightarrow E$ by $S_{1}(\overline{\mathbf{x}}, \overline{\mathbf{y}})=$ $(0, S \overline{\mathbf{x}})$. Clearly $S_{1}$ is a compactly dominated compact operator on the Dedekind complete Banach lattice $E$, and its modulus $\left|S_{1}\right|=(0,|S|)$ is not compact.

Using the previous result we can easily answer question (Q3).

Corollary 2. There exist a pair of compact positive operators $K_{1}, K_{2}$ on a Dedekind complete Banach lattice $E$, such that $K_{1} \vee K_{2}$ is not compact.

Proof. Take the Dedekind complete Banach lattice $E$ and the compact operator $S: E \rightarrow E$ guaranteed by Theorem 1 (ii). Let $U \geq \pm S$ be a compact majorant 
of $S$. If we define $K_{1}=U+S$ and $K_{2}=U-S$, then both of these operators are positive and compact. Evidently we have

$$
K_{1} \vee K_{2}=(U+S) \vee(U-S)=U+S \vee(-S)=U+|S|,
$$

which is not compact.

The example we have given is not, of course, the only one possible. Indeed, using a result in [AJ], the argument we have used can be repeated to show that if $E$ is a Banach lattice which is not isomorphic to an AL-space and $F$ is an appropriate separable rearrangement invariant Banach function space (say any $\left.L_{p}[0,1]\right)$, then there is a compactly dominated compact operator $S: E \rightarrow F$ such that $|S|$ is not compact.

Let us denote by $\mathscr{K}^{r}(E, F)$ the space of all compactly dominated compact operators between Banach lattices $E$ and $F$. In other words, $\mathscr{K}^{r}(E, F)=$ $\mathscr{K}_{+}(E, F)-\mathscr{K}_{+}(E, F)$, where $\mathscr{K}_{+}(E, F)$ denotes, as usual, the cone of compact positive operators. There are two ways of looking at order properties of the space $\mathscr{K}^{r}=\mathscr{K}^{r}(E, F)$. We can study $\mathscr{K}^{r}$ as a vector subspace of $\mathscr{L}^{r}(E, F)$, the space of all regular operators from $E$ into $F$. We can also study $\mathscr{K}^{r}$ as an independent ordered space in its own right, forgetting its natural embedding in $\mathscr{L}^{r}$. The latter approach was undertaken by the second author in [W2], where he found necessary and sufficient conditions on $E$ and $F$ for $\mathscr{K}^{r}(E, F)$ to be a $\sigma$-Dedekind complete (or Dedekind complete) vector lattice.

Although it was not spelled out there, the results in [W2] show (by looking at the three cases separately) that if $\mathscr{K}^{r}(E, F)$ is a Dedekind $\sigma$-complete vector lattice, then for each pair $S, T \in \mathscr{K}^{r}(E, F)$ the supremum calculated in $\mathscr{K}^{r}(E, F)$ is also their supremum in $\mathscr{L}^{r}(E, F)$. Furthermore, the natural embedding of $\mathscr{K}^{r}(E, F)$ into $\mathscr{L}^{r}(E, F)$ will be order continuous. If $\mathscr{K}^{r}(E, F)$ is actually Dedekind complete, then $F$ will be also; consequently $\mathscr{L}^{r}(E, F)$ is a Dedekind complete vector lattice and $\mathscr{K}^{r}(E, F)$ is an order closed sublattice of it. Thus assuming slightly more than just $\mathscr{K}^{r}(E, F)$ being a lattice forces a rather strong connection between $\mathscr{K}^{r}(E, F)$ and $\mathscr{L}^{r}(E, F)$.

In general however, as our next result shows, the space $\mathscr{K}^{r}$ may fail to be a vector lattice even if $F$ is Dedekind complete.

Corollary 3. The operator order on $\mathscr{K}^{r}\left(l_{1}\left(l_{2}^{2^{n}}\right), L_{2}[0,1]\right)$ is not a lattice order. Proof. Take $S \in \mathscr{K}^{r}=\mathscr{K}^{r}\left(l_{1}\left(l_{2}^{2^{n}}\right), L_{2}[0,1]\right)$ constructed in Theorem 1(i). We claim that in $\mathscr{K}^{r}$ there is no modulus of $S$.

Assume, contrary to what we claim, that this modulus exists, and denote it by $|S|_{k}$. Clearly, $|S|_{k} \geq|S|$, where $|S|$ is the usual modulus of $S$ evaluated in $\mathscr{L}^{r}\left(l_{1}\left(l_{2}^{2^{n}}\right), L_{2}[0,1]\right)$. It is also clear that, restricting $|S|_{k}$ and $|S|$ to the order ideal $l_{2}^{2^{n}}$, we have the equality $|S|_{k \mid 2_{2}^{n}}=|S|_{\mid 2_{2}^{n}}$ as the operator on the righthand side is compact. Therefore, for the element $\overline{\mathbf{x}}=\left(0, \ldots, 0, \mathbf{x}_{\mathbf{n}}, 0, \ldots\right)$ constructed in the proof of Theorem 1(i), we have

$$
|S|_{k}(\overline{\mathbf{x}})=|S| \overline{\mathbf{x}}=2^{-n / 2} g_{n} J_{n}\left|S_{n}\right| \mathbf{x}_{\mathbf{n}}=g_{n},
$$

i.e., the image of the unit ball under $|S|_{k}$ contains the sequence $\left\{g_{n}\right\}$, and consequently, $|S|_{k} \in \mathscr{K}^{r}$ cannot be compact, a contradiction.

In connection with Corollary 3 and with the results from [W2] mentioned above, we pose several new problems here. 
(Q4) Is is possible to construct Banach lattices $E, F$ such that $F$ is Dedekind complete and $\mathscr{K}^{r}(E, F)$ is a vector lattice but is not a sublattice of $\mathscr{L}^{r}(E, F)$ ?

If we do not require $F$ be Dedekind complete, then $\mathscr{L}^{r}(E, F)$ may well not be a vector lattice, and the previous question should be slightly modified to make sense.

(Q5) Is it possible to construct Banach lattices $E, F$ such that $\mathscr{K}^{r}(E, F)$ is a vector lattice and there exist $S, T \in \mathscr{K}^{r}(E, F)$ such that $S \vee T$, calculated in $\mathscr{K}^{r}(E, F)$, is not their supremum in $\mathscr{L}^{r}(E, F)$ ?

Our last problem is intermediate between (Q4) and (Q5).

(Q6) Is it possible to construct Banach lattices $E, F$ such that $\mathscr{L}^{r}$ and $\mathscr{K}^{r}$ are both lattices, without the latter being a sublattice of the former?

\section{A COUNTEREXAMPle to (Q1)}

Let us denote $H_{n}=J_{n}\left(l_{2}^{2^{n}}\right)$. This is a $2^{n}$-dimensional vector sublattice of $L_{2}$ order isomorphic to $l_{2}^{2^{n}}$. We denote by $Q_{n}$ the natural projection from $L_{2}$ onto $H_{n}$ defined by

$$
Q_{n}: f \mapsto 2^{n} \sum_{k=1}^{2^{n}}\left(\int_{\frac{k-1}{2^{n}}}^{\frac{k}{2^{n}}} f d \mu\right) \chi_{\left[\frac{k-1}{2^{n}}, \frac{k}{2^{n}}\right]}
$$

Obviously $Q_{n}$ is positive and contractive. Indeed

$$
\begin{aligned}
\left\|Q_{n} f\right\|_{L_{2}}^{2} & =2^{2 n} \sum_{k=1}^{2^{n}} 2^{-n}\left(\int_{\frac{k-1}{2^{n}}}^{\frac{k}{2^{n}}} f d \mu\right)^{2} \leq 2^{n} \sum_{k=1}^{2^{n}}\left(\int_{\frac{k-1}{2^{n}}}^{\frac{k}{2^{n}}}|f| d \mu\right)^{2} \\
& \leq \sum_{k=1}^{2^{n}} \int_{\frac{k-1}{2^{n}}}^{\frac{k}{2^{n}}}|f|^{2} d \mu=\int_{0}^{1}|f|^{2} d \mu=\|f\|_{L_{2}}^{2} .
\end{aligned}
$$

A direct verification shows that for each $n$ and each $f \in L_{2}$ we have

$$
2^{-n} J_{n} \circ\left|S_{n}\right| \circ J_{n}^{-1} \circ Q_{n}(f)=\left(\int_{0}^{1} f d \mu\right) \chi_{[0,1]} \text {. }
$$

Now we are ready to construct a compact, compactly dominated operator $S$ without a modulus. It is curious that this example is simpler than a weaker example constructed in [AW]. However, the idea is similar to that used in [AW]. We denote by $c\left(L_{2}[0,1]\right)$ the Banach lattice of all norm convergent sequences $\left(f_{1}, f_{2}, \ldots\right)$ of functions from $L_{2}$ for which

$$
\left\|\left(f_{1}, f_{2}, \ldots\right)\right\|_{c\left(L_{2}[0,1]\right)}:=\lim _{n \rightarrow \infty}\left\|f_{n}\right\|_{L_{2}}<\infty .
$$

Theorem 4. (i) There exists a compactly dominated compact operator from $L_{2}[0,1]$ to $c\left(L_{2}[0,1]\right)$ without modulus.

(ii) There exists a Banach lattice $E$ and a compactly dominated compact operator on $E$ without modulus.

Proof. (i) Let $\gamma_{n}=\frac{1-(-1)^{n}}{2}, n \in \mathbb{N}$. For each $f \in L_{2}$ we have $J_{n} S_{n} J_{n}^{-1} Q_{n} f \in$ $L_{2}$ and the sequence $2^{-n}\left\|J_{n} S_{n} J_{n}^{-1} Q_{n} f\right\| \rightarrow 0$ as $n \rightarrow \infty$. Therefore, by letting

$$
S f=\left(\gamma_{n} 2^{-n} J_{n} S_{n} J_{n}^{-1} Q_{n} f\right)_{n=1}^{\infty}
$$

we clearly define an operator from $L_{2}$ to $c\left(L_{2}\right)$. This operator $S$ is compact, being the norm limit of finite rank operators. 
Define also $U: L_{2} \rightarrow c\left(L_{2}\right)$ by

$$
U f=\left(\int_{0}^{1} f d \mu\right) \overline{\mathbf{1}},
$$

where $\overline{\mathbf{1}}=\left(\chi_{[0,1]}, \chi_{[0,1]}, \ldots\right)$ is the constantly one sequence in $c\left(L_{2}\right)$. Clearly $U$ is positive and compact as it has rank 1 , and also $\pm S \leq U$.

We claim that operator $S$ does not have a modulus in the space $\mathscr{L}^{r}\left(L_{2}, c\left(L_{2}\right)\right)$ of all regular operators. Assume contrary to what we claim that $|S|=S \vee(-S)$ exists. This implies that $|S| f \geq\left( \pm \gamma_{n} 2^{-n} J_{n} S_{n} J_{n}^{-1} Q_{n} f\right)_{n=1}^{\infty}$ for each $0 \leq f \in L_{2}$, and consequently $|S| f \geq\left(\gamma_{n} 2^{-n} J_{n}\left|S_{n}\right| J_{n}^{-1} Q_{n} f\right)_{n=1}^{\infty}$. Therefore, $|S|\left(\chi_{[0,1]}\right) \geq\left( \pm \gamma_{n} 2^{-n} J_{n}\left|S_{n}\right| J_{n}^{-1} Q_{n} \chi_{[0,1]}\right)_{n=1}^{\infty}$. Notice now that if $n$ is odd, then the $n$th coordinate $\gamma_{n} 2^{-n} J_{n}\left|S_{n}\right| J_{n}^{-1} Q_{n} \chi_{[0,1]}=\chi_{[0,1]}$, whence the norms of the coordinates of the element $|S|\left(\chi_{[0,1]}\right)$ have to be eventually bounded away from zero. However, this is impossible since, by definition, all even coordinates of $S$ are zero, and the same will hold for $|S|$ by a standard argument.

(ii) The proof that (ii) follows from (i) is similar to the proof of the implication (i) $\Rightarrow$ (ii) in Theorem 1 .

Remark. The same proof shows that the operator $S$ constructed above does not have a modulus in the space $\mathscr{K}^{r}(E)$ either.

A discussion similar to that after Corollary 2 shows that we can generalize the constructions of Theorem 4 and produce various analogous "abstract" examples.

\section{REFERENCES}

[AJ] Y. A. Abramovich and L. P. Janovsky, Applications of the Rademacher systems to operator characterizations of Banach lattices, Colloq. Math. 46 (1982), 75-78.

[AW] Y. A. Abramovich and A. W. Wickstead, A compact regular operator without modulus, Proc. Amer. Math. Soc. 116 (1992), 721-726.

[AB] C. D. Aliprantis and O. Burkinshaw, Positive operators, Academic Press, New York and London, 1985.

[DF] P. G. Dodds and D. H. Fremlim, Compact operators in Banach lattices, Israel J. Math. 34 (1979), 287-320.

[HL] C. B. Huijsmans and W. A. J. Luxemburg, eds., Positive operators and semigroups on Banach lattices, Kluwer, Dordrecht, 1992.

[K1] U. Krengel, Remark on the modulus of compact operators, Bull. Amer. Math. Soc. 72 (1966), 132-133.

[K2] _ Über den Absolutbetrag stetiger linearer Operatoren und seine Anwendung auf ergodische Zerlegung, Math. Scand. 13 (1963), 151-187.

[W1] A. W. Wickstead, Converses for the Dodds-Fremlin and Kalton-Saab theorems, Math. Proc. Cambridge Philos. Soc. (to appear).

[W2] __ Dedekind completeness of some lattices of compact operators, Bull. Polish. Acad. Sci. Math. (to appear).

Department of Mathematics, Indiana University/ Purdue University, Indianapolis, INDIANA 46202

E-mail address: yabramovich@math.iupui.edu

Department of Pure Mathematics, The Queen's University of Belfast, Belfast BT7 INN, NoRTHERN IRELAND

E-mail address: A.Wickstead@qub.ac.uk 